\title{
ON LOWER AND UPPER SOLUTIONS WITHOUT ORDERING ON TIME SCALES
}

PETR STEHLÍK

Received 31 January 2006; Revised 16 May 2006; Accepted 16 May 2006

In order to enlarge the set of boundary value problems on time scales, for which we can use the lower and upper solutions technique to get existence of solutions, we extend this method to the case when the pair lacks ordering. We use the degree theory and a priori estimates to obtain the existence of solutions for the second-order Dirichlet boundary value problems. To illustrate a wider application of this result, we conclude with an example which shows that a combination of well- and nonwell- ordered pairs can yield the existence of multiple solutions.

Copyright (C 2006 Petr Stehlík. This is an open access article distributed under the Creative Commons Attribution License, which permits unrestricted use, distribution, and reproduction in any medium, provided the original work is properly cited.

\section{Introduction}

The method of lower and upper solutions is a widely used concept in the study of nonlinear boundary value problems (further abbreviated by BVP). Three quarters of the century after the pioneering work of Dragoni [8] this method still belongs among the basic tools and is frequently employed in applied analysis or mechanics. Dragoni's basic idea was to transform the BVP with an unbounded right-hand side into a problem with a bounded right-hand side (this transformation is possible thanks to the existence of lower and upper solutions) and, in the second step, to show that a solution of the modified problem is also a solution of the original problem. Together with the later introduced Nagumo conditions for the derivative dependent right-hand sides this basic scheme forms the foundations of this method.

On the other hand, the time scales calculus, with its concept to unify and extend discrete and continuous worlds, is a recent idea (the seminal work is due to Hilger, see, e.g., [9]). In spite of this, this calculus is already broadly used. It is not surprising that, after the Schauder fixed point theorem for bounded right-hand sides, the lower and upper solutions technique was used to investigate the problems with unbounded right-hand sides. The first results for Dirichlet boundary conditions are due to Akin [2], or Bohner 
and Peterson [3, Section 6.6]. Later, similar statements were obtained also for periodic conditions, see, for example, Cabada [5], Stehlík [12], or Topal [13].

The main drawback of the concept of lower and upper solutions, which often hinders its practical application, is the assumption on their existence. Logical reaction to this objection was a successful attempt to include also the case when the lower and upper solutions do not satisfy the common ordering, that is, the lower solution is above the upper solution in some points of the considered interval. The so-called nonwell-ordered case for differential equations was first studied in 1970s, see, for example, Sattinger [11].

The traditional ways to deal with the nonwell-ordered pairs rely on the periodicity and boundedness of trigonometric functions, properties of Fučík spectrum and the existence of intersections of lower and upper solutions (for the survey on lower and upper solutions, see, e.g., De Coster and Habets [6]). Unfortunately, one cannot straightforwardly extend these concepts to the discrete or time scales context. Therefore, we avoid these approaches by relying on the degree theory.

We recall the basic definitions and notations concerning time scales calculus, the reader acquainted with the basic concepts (within the scope of the first chapters of Bohner and Peterson $[3,4])$ can jump over to (1.6).

Time scale $\mathbb{T}$ is an arbitrary nonempty closed subset of the real numbers $\mathbb{R}$. The natural numbers $\mathbb{N}$, the integers $\mathbb{Z}$, or the union of intervals $[0,1] \cup[2,3]$ are the most natural examples.

For $t \in \mathbb{T}$ we define the forward jump operator $\sigma: \mathbb{T} \rightarrow \mathbb{T}$ and the backward jump operator $\varrho: \mathbb{T} \rightarrow \mathbb{T}$ by

$$
\varrho(t):=\inf \{s \in \mathbb{T}: s>t\}, \quad \varrho(t):=\sup \{s \in \mathbb{T}: s<t\},
$$

where we put inf $\varnothing=\sup \mathbb{T}$ and $\sup \varnothing=\inf \mathbb{T}$. We say that a point $t \in \mathbb{T}$ is right-scattered, left-scattered, right-dense, left-dense if $\sigma(t)>t, \varrho(t)<t, \sigma(t)=t, \varrho(t)=t$, respectively. Moreover, we define the forward graininess function $\mu: \mathbb{T} \rightarrow[0, \infty)$ by

$$
\mu(t):=\sigma(t)-t
$$

In the above references one can find the definition of the so-called delta-derivative $x^{\Delta}$, which is equivalent to $x^{\prime}$ if $\mathbb{T}=\mathbb{R}$, or to $\Delta x$ if $\mathbb{T}=\mathbb{Z}$. Similarly, several concepts of integration have been extended as well, ranging from Cauchy-Newton [3, Section 1.4] to Henstock-Kurzweil [10] integration.

For the sake of clarity we introduce the closed time scale interval by

$$
[a, b]_{\mathbb{T}}=[a, b] \cap \mathbb{T},
$$

with the note that other types of intervals are defined in the analogous way.

To simplify complicated formulae, we use the abridged notations

$$
x^{\sigma}(t)=x(\sigma(t)), \quad x^{\rho}(t)=x(\varrho(t)) .
$$


We define an rd-continuous function as a function that is continuous in all right-dense points and left-sided limits exist in left-dense points. The set of all rd-continuous functions will be denoted by $C_{\text {rd }}$. The set of twice differentiable functions whose second derivative is rd-continuous will be denoted by $C_{\mathrm{rd}}^{2}$. Finally, we define the following function space:

$$
C_{\mathrm{rd}, 0}^{2}\left(\left[0, \sigma^{2}(1)\right]_{\mathbb{T}}\right):=\left\{x \in C_{\mathrm{rd}}^{2}: x(0)=x\left(\sigma^{2}(1)\right)=0\right\}
$$

For the sake of brevity, we often use $C_{\mathrm{rd}, 0}^{2}$ instead.

In this paper we consider a nonwell-ordered couple of lower and upper solutions for the following Dirichlet BVP:

$$
\begin{gathered}
-x^{\Delta \Delta}(t)=f\left(t, x^{\sigma}(t)\right) \quad \text { on }[0,1]_{\mathbb{T}} \\
x(0)=x\left(\sigma^{2}(1)\right)=0 .
\end{gathered}
$$

The solution of (1.6) is a function $x \in C_{\mathrm{rd}, 0}^{2}$ which satisfies the equation for all $t \in[0,1]_{\mathbb{T}}$.

We base our work on the existence theorems for the well-ordered case which are presented in Akin [2]. Therefore, we start, in Section 2, with a slight modification of one of these results. Namely, we provide further information about the degree of the corresponding operator.

Next, in Section 3, we use this extension to prove the existence in nonwell-ordered setting. Aside from the degree theory, a priori estimate and the properties of first eigenvalue and eigenfunction are our main tools. If $f$ satisfies certain growth and limit conditions, we obtain the existence of a solution. The similar approach for the $p$-Laplacian can be found in Drábek et al. [7].

Finally, by combining these results we suggest how to acquire the existence of multiple solutions. This idea is illustrated, in Section 4, on the existence of three solutions.

\section{Well-ordered case}

In this section we present the basic definitions and notations for lower and upper solutions and we amend the existing results for well-ordered pairs. Let us first define lower and upper solutions for BVP (1.6).

Definition 2.1. A function $\alpha \in C_{\mathrm{rd}}^{2}\left(\left[0, \sigma^{2}(1)\right]_{\mathbb{\Psi}}\right)$ is called a lower solution of (1.6) if

$$
\begin{gathered}
\alpha(0) \leq 0, \quad \alpha\left(\sigma^{2}(1)\right) \leq 0, \\
-\alpha^{\Delta \Delta}(t) \leq f\left(t, \alpha^{\sigma}\right) \quad \forall t \in\left[0, \sigma^{2}(1)\right]_{\mathbb{T}} .
\end{gathered}
$$

Similarly, a function $\beta \in C_{\mathrm{rd}}^{2}\left(\left[0, \sigma^{2}(1)\right]_{\mathbb{T}}\right)$ is called an upper solution of (1.6) if

$$
\begin{gathered}
\beta(0) \geq 0, \quad \beta\left(\sigma^{2}(1)\right) \geq 0, \\
-\beta^{\Delta \Delta}(t) \geq f\left(t, \beta^{\sigma}\right) \quad \forall t \in\left[0, \sigma^{2}(1)\right]_{\mathbb{T}} .
\end{gathered}
$$

Next, let us define the ordering in $C_{\mathrm{rd}, 0}^{2}$. 
4 Dynamic lower and upper solutions without ordering

Definition 2.2. A function $x$ is strictly smaller than $y$ (denoted by $x \ll y$ ) if

$$
x(t)<y(t) \quad \text { for } t \in\left(0, \sigma^{2}(1)\right)_{\mathbb{T}},
$$

and the following conditions hold on the boundary:

(i) either $x(0)<y(0)$, or $x^{\Delta}(0)<y^{\Delta}(0)$, and

(ii) either $x\left(\sigma^{2}(1)\right)<y\left(\sigma^{2}(1)\right)$, or $x^{\Delta}(\sigma(1))<y^{\Delta}(\sigma(1))$.

Using this ordering we can define an important subclass of lower and upper solutions. Definition 2.3. A function $\alpha$ is a strict lower solution of (1.6) if

(i) $\alpha$ is a lower solution of (1.6),

(ii) every possible solution $x$ of (1.6) satisfying $\alpha \leq x$ satisfies $\alpha \ll x$.

Reversing the above inequality we can get the corresponding definition of a strict upper solution.

As usual, we introduce the solution operator $T: C_{\mathrm{rd}, 0}^{2} \rightarrow C_{\mathrm{rd}, 0}^{2}$ defined by

$$
T x(t):=\int_{0}^{\sigma(1)} G(t, s) f\left(s, x^{\sigma}(s)\right) \Delta s
$$

where $G(t, s)$ is a Green's function for (1.6) with $f(t, s)=0$ (see, e.g., [3, Corollary 4.76]).

At this stage, we are ready to state an expanded existence theorem.

Theorem 2.4. Let $f$ be a continuous function. Let $\alpha, \beta$ be lower and upper solutions, respectively, for which $\alpha \leq \beta$ holds. Then the problem (1.6) has at least one solution $x$ satisfying

$$
\alpha \leq x \leq \beta \quad \text { in }\left[0, \sigma^{2}(1)\right]_{\mathbb{T}} .
$$

Furthermore, if $\alpha$ and $\beta$ are strict and $\alpha \ll \beta$ holds, then there exists $R_{0}>0$, such that for all $R>R_{0}$

$$
\operatorname{deg}\left(I-T ; \Omega_{1}, o\right)=1
$$

where

$$
\Omega_{1}:=\left\{x \in C_{\mathrm{rd}}^{2}\left(\left[0, \sigma^{2}(1)\right]_{\mathbb{T}}\right): \alpha \ll x \ll \beta\right\} \cap B(o, R) .
$$

Proof. With the purpose of applying the Schauder fixed point theorem, Bohner and Peterson, in [3, Theorem 6.54], define a modified right-hand side function by

$$
\hat{f}(t, x)= \begin{cases}f\left(t, \beta^{\sigma}(t)\right)+\frac{x-\beta^{\sigma}(t)}{1+|x|} & \text { if } x \geq \beta^{\sigma}(t) \\ f(t, x) & \text { if } \alpha^{\sigma}(t) \leq x \leq \beta^{\sigma}(t), \\ f\left(t, \alpha^{\sigma}(t)\right)+\frac{x-\beta^{\sigma}(t)}{1+|x|} & \text { if } x \leq \alpha^{\sigma}(t) .\end{cases}
$$

Using the continuity and boundedness of this function, it obtains the compactness of $\widehat{T}$ (defined by (2.4) with $f$ replaced by $\hat{f}$ ) and, consequently, fixed point $x$ of this modified 
operator. Finally, one can show that $\alpha(t) \leq x(t) \leq \beta(t)$ which implies that $x$ is also the fixed point of $T$ defined by (2.4) and thus a solution of (1.6).

We define the constant $R_{0}>0$ as a bound of operator $\hat{T}$ (the existence of this bound is ensured by the definition of $\hat{f}$ ), that is, for each $y \in C_{\mathrm{rd}, 0}^{2}$ we have

$$
\|\hat{T}(y)\|_{C_{\mathrm{rd}, 0}^{2}}<R_{0} .
$$

This guarantees the existence of an admissible homotopy:

$$
H(\tau, x)=I(x)-\tau \widehat{T}(x) \quad \tau \in[0,1]
$$

which implies the following equality of degrees:

$$
\operatorname{deg}\left(I-\widehat{T} ; B\left(o, R_{0}\right), o\right)=\operatorname{deg}\left(I ; B\left(o, R_{0}\right), o\right)=1 .
$$

Moreover, since $\alpha$ and $\beta$ are strict, there is no solution of $x=\hat{T}(x)$ with $x(t) \leq \alpha(t)$ or $x(t) \geq \beta(t)$ for any $t \in\left(0, \sigma^{2}(1)\right)_{\mathbb{T}}$ and we can deduce that

$$
\operatorname{deg}\left(I-\widehat{T} ; \Omega_{1}, o\right)=\operatorname{deg}\left(I-\widehat{T} ; B\left(o, R_{0}\right), o\right)=1 .
$$

To conclude, the definition of $\Omega_{1}$ yields that

$$
\operatorname{deg}\left(I-T ; \Omega_{1}, o\right)=\operatorname{deg}\left(I-\widehat{T} ; \Omega_{1}, o\right)=1 .
$$

\section{Nonwell-ordered case}

First of all, we recall the basic results concerning the eigenvalue problem:

$$
\begin{gathered}
-x^{\Delta \Delta}(t)=\lambda x(t) \quad \text { on }\left[0, \sigma^{2}(1)\right]_{\mathbb{T}}, \\
x(0)=x\left(\sigma^{2}(1)\right)=0 .
\end{gathered}
$$

Using the existing oscillation theorem we can prove this simple statement.

Lemma 3.1. The first eigenvalue $\lambda_{1}$ of (3.1) is positive and the corresponding eigenfunction $\varphi_{1}(t)>0$ for all $t \in\left(0, \sigma^{2}(1)\right)_{\mathbb{T}}$.

Proof. Obviously, (3.1) has only a trivial solution if $\lambda=0$. Now, let us suppose that $\lambda<0$ is an eigenvalue. The corresponding eigenfunction $\varphi(t)$ (or $-\varphi(t))$ must attain a maximum in $\left(0, \sigma^{2}(1)\right)_{\mathbb{T}}$. Let us suppose that $m \in\left(0, \sigma^{2}(1)\right)_{\mathbb{T}}$ is the first point where the maximum is attained. Let us distinguish between two cases.

(i) $m$ is left-dense. In that case $\varphi^{\Delta \Delta}(m) \leq 0$ and $\varphi^{\Delta}(m)=0$, which leads to the following contradiction:

$$
0 \geq \varphi^{\Delta \Delta}(m)=\lambda \varphi^{\sigma}(m)=\lambda\left(\varphi(m)+\mu(m) \varphi^{\Delta}(m)\right)=\lambda \varphi(m)>0 .
$$


6 Dynamic lower and upper solutions without ordering

(ii) $m$ is left-scattered. This implies that $x^{\Delta}(m) \leq 0$ and $x^{\Delta}(\varrho(m))>0$ and we can reach a contradiction by

$$
0<\lambda \varphi(m)=\varphi^{\Delta \Delta}(\varrho(m))=\frac{\varphi^{\Delta}(m)-\varphi^{\Delta}(\varrho(m))}{\mu^{\rho}(m)}<0 .
$$

The positivity of first eigenfunctions is the immediate consequence of oscillation theorem, which is due to Agarwal et al. [1, Theorem 1] or Bohner and Peterson [3, Theorem 4.106].

At this stage, we are ready to prove the existence result also in the case when lower and upper solutions are without ordering.

THEOREM 3.2. Let $f$ be a continuous function satisfying that

(i) there are $c, d>0$ such that

$$
|f(t, s)| \leq c+d|s|
$$

for all $t \in[0,1]_{\mathbb{T}}$ and for all $s \in \mathbb{R}$, and

(ii)

$$
\lim _{|s| \rightarrow \infty} \frac{f(t, s)}{s}=\lambda_{1}
$$

Assume that $\alpha, \beta$ are lower and upper solutions and that there exists $\tau \in\left(0, \sigma^{2}(1)\right)_{\mathbb{T}}$ such that

$$
\alpha(\tau)>\beta(\tau)
$$

Then (1.6) has at least one solution in

$$
S:=\overline{\left\{x \in C_{\mathrm{rd}, 0}^{2}: \exists \zeta, \eta \in\left(0, \sigma^{2}(1)\right)_{\mathbb{T}}: x(\zeta)<\alpha(\zeta), x(\eta)>\beta(\eta)\right\}} C_{\mathrm{rd}, 0}^{2} .
$$

If we define $\Omega_{2}:=S \cap B(o, R)$ and assume that there is no solution on $\partial \Omega_{2}$, then there exists $R_{0}>0$ such that for all $R>R_{0}$ :

$$
\operatorname{deg}\left(I-T ; \Omega_{2}, o\right)=-1
$$

Proof. We assume that there is no solution on $\partial S$ (otherwise there is no reason to proceed with the proof). For the sake of lucidity, we divide our proof into three parts.

(i) A priori estimate. First, let us prove that if we define $f_{r}:[0,1]_{\mathbb{T}} \times \mathbb{R} \rightarrow \mathbb{R}$ by

$$
f_{r}(t, y):= \begin{cases}f(t, y) & \text { if }|y|<r \\ (1+r-|y|) f(t, y) & \text { if } r \leq|y| \leq r+1 \\ 0 & \text { if }|y| \geq r+1\end{cases}
$$


then there exists $K>0$ such that for any $r>0$ and any solution $x \in S$ of

$$
\begin{gathered}
-x^{\Delta \Delta}(t)=f_{r}\left(t, x^{\sigma}(t)\right) \quad \text { on }[0,1]_{\mathbb{T}}, \\
x(0)=x\left(\sigma^{2}(1)\right)=0,
\end{gathered}
$$

the following a priori estimate holds:

$$
\|x\|_{C_{\mathrm{rd}, 0}^{2}} \leq K
$$

As usual, we suppose that this assumption is not satisfied, that is, there exists a sequence $\left\{r_{k}\right\}_{k=1}^{\infty}$ with $r_{k}>0$ and a corresponding sequence of solutions $\left\{x_{k}\right\}_{k=1}^{\infty}$ satisfying $\left\|x_{k}\right\|_{C_{\mathrm{rd}, 0}^{2}} \geq k$ and solving

$$
\begin{gathered}
-x_{k}^{\Delta \Delta}(t)=f_{r_{k}}\left(t, x_{k}^{\sigma}(t)\right) \quad \text { on }[0,1]_{\mathbb{T}}, \\
x_{k}(0)=x_{k}\left(\sigma^{2}(1)\right)=0 .
\end{gathered}
$$

Defining $y_{k}:=x_{k} /\left\|x_{k}\right\|_{C_{\mathrm{rd}, 0}^{2}}$ and dividing (3.12) by $\left\|x_{k}\right\|_{C_{\mathrm{rd}, 0}^{2}}$ we obtain

$$
\begin{gathered}
-y_{k}^{\Delta \Delta}(t)=\frac{f_{r_{k}}\left(t, x_{k}^{\sigma}(t)\right)}{\left\|x_{k}\right\|_{C_{\mathrm{rd}, 0}^{2}}} \text { on }[0,1]_{\mathbb{T}}, \\
y_{k}(0)=y_{k}\left(\sigma^{2}(1)\right)=0 .
\end{gathered}
$$

The boundedness of the sequence (clearly $\left\|y_{k}\right\|_{C_{\mathrm{rd}, 0}^{2}}=1$ ) and the compactness of $T$ provide convergence (at least for a subsequence) to some $y \in C_{\mathrm{rd}, 0}^{2}$

$$
y_{k} \rightarrow y \quad \text { in } C_{\mathrm{rd}, 0}^{2} \text {. }
$$

The condition (3.4) implies that for some sufficiently large $\varepsilon \geq 0$ the right-hand sides of (3.13) are bounded by a constant (and thus integrable) function $h(s)=d+\varepsilon$ and, moreover, using the limit assumption (3.5) we can get

$$
\frac{f_{r_{k}}\left(t, x_{k}^{\sigma}(t)\right)}{\left\|x_{k}\right\|_{C_{\mathrm{rd}, 0}^{2}}^{2}}=\frac{f_{r_{k}}\left(t, x_{k}^{\sigma}(t)\right)}{\left\|x_{k}\right\|_{C_{\mathrm{rd}, 0}^{2}}} \frac{x_{k}}{x_{k}} \longrightarrow \lambda_{1} y .
$$

Thus the dominated convergence theorem (see Peterson, Thompson [10, Theorem 2.17] for its most general form on time scales) yields that $y$ solves the problem:

$$
\begin{gathered}
-y^{\Delta \Delta}(t)=\lambda_{1} y(t) \quad \text { on }[0,1]_{\mathbb{T}}, \\
y(0)=y\left(\sigma^{2}(1)\right)=0 .
\end{gathered}
$$

Taking into account $\|y\|=1$ again we obtain that $y$ is a nonzero multiple of the first eigenfunction $\varphi(t)>0$ for $t \in\left(0, \sigma^{2}(1)\right)_{\mathbb{T}}$ (see Lemma 3.1). If $y$ is positive, then $x_{k}(t) \rightarrow \infty$ for all $t \in\left(0, \sigma^{2}(1)\right)_{\mathbb{T}}$ which implies that there is $k_{0} \in \mathbb{N}$ such that for all $k>k_{0}$ there does not exist $\xi \in\left(0, \sigma^{2}(1)\right)_{\mathbb{T}}$ such that the inequality $x_{k}(\xi)<\alpha(\xi)$ holds. Therefore $x_{k} \notin S$, a contradiction. 
8 Dynamic lower and upper solutions without ordering

Similarly, if $y$ is negative, then there is $k \in \mathbb{N}$ such that for all $k>k_{0}$ and for all $\eta \in$ $\left(0, \sigma^{2}(1)\right)_{\mathbb{T}}$ we have $x(\eta)<\beta(\eta)$, a contradiction.

(ii) Construction of strict well-ordered lower and upper solutions. Let us consider an arbitrary $R>0$ satisfying

$$
R>R_{0}:=\max \left\{K,\|\alpha\|_{C},\|\beta\|_{C}\right\}+1
$$

and the BVP (3.10) with $r=R$, that is,

$$
\begin{gathered}
-x^{\Delta \Delta}(t)=f_{R}\left(t, x^{\sigma}(t)\right) \quad \text { on }[0,1]_{\mathbb{T}}, \\
x(0)=x\left(\sigma^{2}(1)\right)=0 .
\end{gathered}
$$

We show that $u:=-R-2$ and $v:=R+2$ are lower and upper solutions of (3.18), respectively. Obviously, $u$ is a lower solution since

$$
\begin{gathered}
u(0)=-R-2<0, \quad u\left(\sigma^{2}(1)\right)=-R-2<0, \\
u^{\Delta \Delta}(t)=0=f_{R}(t,-R-2), \quad \forall t \in[0,1]_{\mathbb{T}} .
\end{gathered}
$$

Now assume that $u$ is not strict, that is, there exists $m \in\left(0, \sigma^{2}(1)\right)_{\mathbb{T}}$ defined by

$$
m:=\min _{t \in\left(0, \sigma^{2}(1)\right)_{\mathbb{T}}}\{t: x(t)=-R-2\} .
$$

Again, we divide our reasoning into two parts.

(a) Let us suppose that $m$ is left-dense. The minimality of $x$ at $m$ implies that $x^{\Delta}(m)=$ 0 and the left-density of $m$ provides the existence of $\varepsilon>0$ such that $x(t)<-R-1$ for all $t \in(m-\varepsilon, m)_{\mathbb{T}}$, that is, $f_{R}\left(t, x^{\sigma}(t)\right)=0$ for these $t$. These two facts suggest that $x^{\Delta \Delta}(t)=0$ for all $t \in[m, 1]_{\mathbb{T}}$. Thus $x\left(\sigma^{2}(1)\right)=-R-2$ and $x$ is not a solution of (3.18), a contradiction.

(b) Now, we assume that $m$ is left-scattered. Since $x$ achieves its minimum first at $m$, we obtain the following two conditions:

$$
x^{\Delta}(m) \geq 0, \quad x^{\Delta}(\varrho(m))<0 .
$$

But this leads to the following contradiction:

$$
0=f_{R}(\varrho(m), x(m))=x^{\Delta \Delta}(\varrho(m))=\frac{x^{\Delta}(m)-x^{\Delta}(\varrho(m))}{\mu(\varrho(m))}>0 .
$$

Similarly, one can derive that $v=R+2$ is a strict supersolution of (3.18).

(iii) Computation of the degree. We define $T_{R}: C_{\mathrm{rd}, 0}^{2} \rightarrow C_{\mathrm{rd}, 0}^{2}$ by

$$
T_{R}(x):=\int_{0}^{\sigma(1)} G(t, s) f_{R}\left(s, x^{\sigma}(s)\right)
$$

Obviously, if we find a fixed point $x_{0}$ of $T_{R}$, then $x_{0}$ is a solution of (3.18). If, moreover, $x_{0} \in B(o, R)$, then $x_{0}$ is a solution of $(1.6)$ as well since, thanks to the definition of $f_{R}$, 


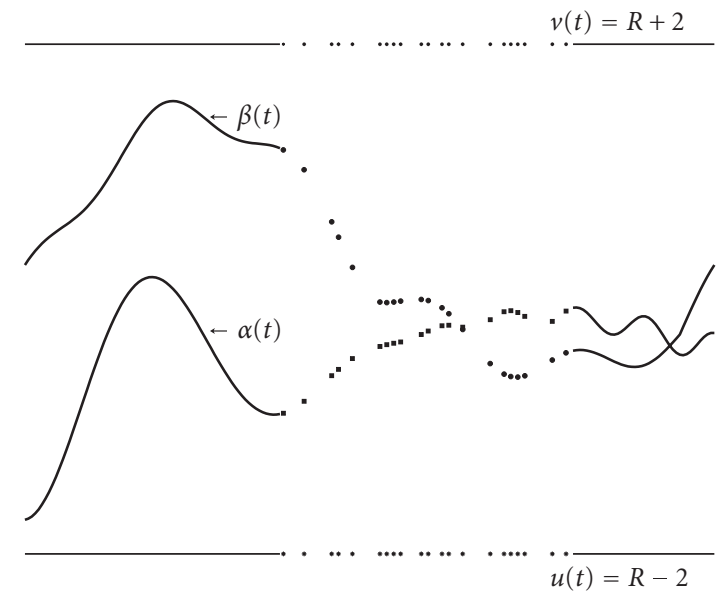

Figure 3.1. Nonwell-ordered case.

operators $T$ and $T_{R}$ coincide on this ball. We define three sets by

$$
\begin{gathered}
\Omega_{u}^{v}:=\left\{x \in C_{\mathrm{rd}, 0}^{2}: u \ll x \ll v\right\}, \\
\Omega_{\alpha}^{v}:=\left\{x \in C_{\mathrm{rd}, 0}^{2}: \alpha \ll x \ll v\right\}, \quad \Omega_{u}^{\beta}:=\left\{x \in C_{\mathrm{rd}, 0}^{2}: u \ll x \ll \beta\right\} .
\end{gathered}
$$

Clearly, $\Omega_{\alpha}^{v}, \Omega_{u}^{\beta}$, and $\Omega_{2}$ are pairwise disjoint subsets. Thus the properties of the degree and the well-ordered result (Theorem 2.4) enable the following computation:

$$
\begin{aligned}
1 & =\operatorname{deg}\left(I-T_{R} ; B(o, R) \cap \Omega_{u}^{v}, o\right) \\
& =\operatorname{deg}\left(I-T_{R} ; B(o, R) \cap \Omega_{u}^{\beta}, o\right)+\operatorname{deg}\left(I-T_{R} ; B(o, R) \cap \Omega_{\alpha}^{v}, o\right)+\operatorname{deg}\left(I-T_{R} ; \Omega_{2}, o\right) \\
& =2+\operatorname{deg}\left(I-T_{R} ; \Omega_{2}, o\right) .
\end{aligned}
$$

Therefore ( $T$ and $T_{R}$ coincide on $B(o, R)$ ), we obtain the required result:

$$
\operatorname{deg}\left(I-T ; \Omega_{2}, o\right)=-1 .
$$

The statement of Theorem 3.2 and the strict pair $u$ and $v$ from its proof are illustrated in Figure 3.1.

Remark 3.3. In contrast to Theorem 2.4, Theorem 3.2 gives less transparent information about the existing solution. This is mainly due to the opaque structure of $S$ (and consequently of $\Omega_{2}$ ). In fact, we know only that there exists a bound $R$ on the norm of this solution $\left(R\right.$ is closer specified in the proof in (3.17)) and that there exist $\xi, \eta \in\left(0, \sigma^{2}(1)\right)_{\mathbb{T}}$ such that $x(\xi)<\alpha(\xi)$ and $x(\eta)>\beta(\eta)$, respectively.

With respect to this remark, we add one simple example to illustrate the above statement. 
Example 3.4. Let us deal with $\mathbb{T}=(1 / 5) \mathbb{Z}$ and a continuous function

$$
\tilde{f}(t, y)= \begin{cases}\lambda_{1}(y+5) & \text { if } y \leq-5 \\ -y(y-5)(y+5) & \text { if }-5<y<5 \\ \lambda_{1}(y-5) & \text { if } y \geq 5\end{cases}
$$

Clearly, $\tilde{f}$ satisfies conditions (i) and (ii) of Theorem 3.2. Let us consider the corresponding BVP:

$$
\begin{gathered}
-x^{\Delta \Delta}(t)=\tilde{f}\left(t, x^{\sigma}(t)\right) \quad \text { on }[0,1]_{\mathbb{T}} \\
x(0)=x\left(\frac{7}{5}\right)=0 .
\end{gathered}
$$

It is easy to verify that $\alpha$ and $\beta$ defined by

\begin{tabular}{l|rrrrrrrr}
\hline$t$ & 0 & $\frac{1}{5}$ & $\frac{2}{5}$ & $\frac{3}{5}$ & $\frac{4}{5}$ & 1 & $\frac{6}{5}$ & $\frac{7}{5}$ \\
\hline$\alpha(t)$ & -1 & $-\frac{3}{2}$ & $-\frac{1}{2}$ & 1 & 2 & $\frac{3}{2}$ & 0 & $-\frac{3}{2}$ \\
$\beta(t)$ & 1 & $\frac{1}{2}$ & $-\frac{1}{2}$ & $-\frac{3}{2}$ & $-\frac{3}{2}$ & $-\frac{1}{2}$ & $\frac{1}{2}$ & 1 \\
\hline
\end{tabular}

are lower and upper solutions of (3.28). Obviously, for all $t \in[3 / 5,1]_{\mathbb{T}}$ we have $\alpha(t)>$ $\beta(t)$. Therefore, we can apply Theorem 3.2 and claim that the problem (3.28) has a solution $x \in C_{\mathrm{rd}, 0}^{2}$. The only additional information about this solution is that there exist $\xi, \eta \in[1 / 5,6 / 5]_{\mathbb{T}}$ such that $x(\xi) \leq \alpha(\xi)$ and $x(\eta) \geq \beta(\eta)$.

\section{Multiple solutions}

The combination of the results for well-ordered case and nonwell-ordered counterpart opens the way for the existence of multiple solutions. As an example of such a process we state a simple result for the existence of three solutions, which can be generalized to other cases.

Theorem 4.1. Let $f$ satisfy the assumptions (i) and (ii) from Theorem 3.2. Assume that $\alpha_{1}$ and $\alpha_{2}$ are lower solutions and $\beta_{1}$ and $\beta_{2}$ are upper solutions of (1.6) which satisfy

$$
\alpha_{1} \ll \beta_{1}, \quad \alpha_{2} \ll \beta_{2},
$$

and assume that there exist $\tau \in\left(0, \sigma^{2}(1)\right)_{\mathbb{T}}$ such that

$$
\alpha_{2}(\tau)>\beta_{1}(\tau)
$$

Then the problem (1.6) has at least three distinct solutions. 


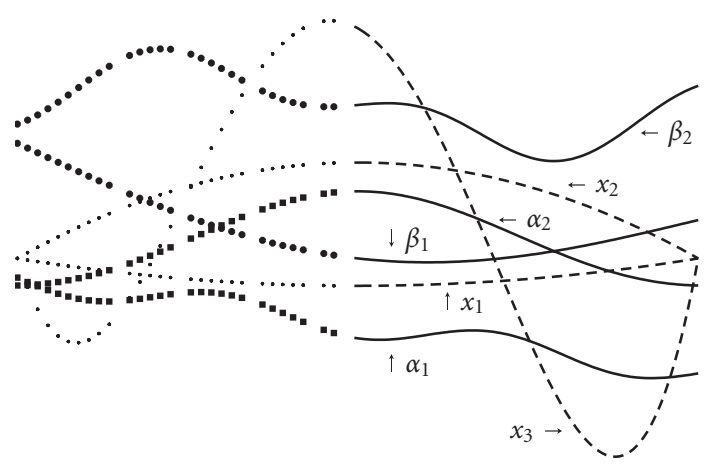

Figure 4.1. Existence of three solutions.

Proof. First, we apply twice Theorem 2.4 to obtain the existence of solutions $x_{1}$ and $x_{2}$ satisfying

$$
\alpha_{1} \ll x_{1} \ll \beta_{1}, \quad \alpha_{2} \ll x_{2} \ll \beta_{2} .
$$

Furthermore, (4.2) allows us to apply Theorem 3.2 to obtain the existence of a solution $x_{3}$. Since $x_{3} \in S$, we know that there exists $\xi, \eta \in\left(0, \sigma^{2}(1)\right)_{\mathbb{T}}$ such that $x_{3}(\xi) \leq \alpha_{2}(\xi)$ and $x_{3}(\eta) \geq \beta_{1}(\eta)$ and this implies that $x_{3}$ is different to $x_{1}, x_{2}$.

The statement of Theorem 4.1 and the solutions $x_{i}, i=\{1,2,3\}$, from its proof are illustrated in Figure 4.1.

Theorem 4.1 basically claims that if you have a nonwell-ordered couple and for each function from this pair you are able to find a function with which it forms an ordered couple, you have at least three solutions. We illustrate this idea on the extension of Example 3.4 .

Example 4.2. Let us assume that $\tilde{f}, \alpha$, and $\beta$ are defined as in Example 3.4. Obviously $\underline{\alpha}(t) \equiv-5$ and $\bar{\beta}(t) \equiv 5$ are another lower and upper solutions of (3.28) (note that $\tilde{f}(t, \pm 5)=0)$. Thus we can claim that the problem (3.28) has at least three distinct so-

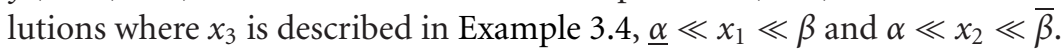

\section{Acknowledgments}

The author gratefully acknowledges the support of the Ministry of Education, Youth and Sports of the Czech Republic, Research Plan no. MSM 4977751301. Moreover, he is also obliged to Pavel Drábek, Bevan Thompson, and Milan Tvrdý for their valuable hints and suggestions.

\section{References}

[1] R. P. Agarwal, M. Bohner, and P. J. Y. Wong, Sturm-Liouville eigenvalue problems on time scales, Applied Mathematics and Computation 99 (1999), no. 2-3, 153-166.

[2] E. Akin, Boundary value problems for a differential equation on a measure chain, Panamerican Mathematical Journal 10 (2000), no. 3, 17-30. 


\section{Dynamic lower and upper solutions without ordering}

[3] M. Bohner and A. Peterson, Dynamic Equations on Time Scales. An Introduction with Applications, Birkhäuser Boston, Massachusetts, 2001.

[4] M. Bohner and A. Peterson (eds.), Advances in Dynamic Equations on Time Scales, Birkhäuser Boston, Massachusetts, 2003.

[5] A. Cabada, Extremal solutions and Green's functions of higher order periodic boundary value problems in time scales, Journal of Mathematical Analysis and Applications 290 (2004), no. 1, 35-54.

[6] C. De Coster and P. Habets, The lower and upper solutions method for boundary value problems, Handbook of Differential Equations (A. Cañada, P. Drábek, and A. Fonda, eds.), Elsevier/NorthHolland, Amsterdam, 2004, pp. 69-160.

[7] P. Drábek, P. Girg, and R. Manásevich, Generic Fredholm alternative-type results for the one dimensional p-Laplacian, Nonlinear Differential Equations and Applications 8 (2001), no. 3, 285298.

[8] G. S. Dragoni, II problema dei valori ai limiti studiato in grande per le equazioni differenziali del secondo ordine, Mathematische Annalen 105 (1931), no. 1, 133-143.

[9] S. Hilger, Analysis on measure chains - a unified approach to continuous and discrete calculus, Results in Mathematics 18 (1990), no. 1-2, 18-56.

[10] A. Peterson and H. B. Thompson, The Henstock-Kurzweil delta and nabla integrals, to appear in Journal of Mathematical Analysis and Applications.

[11] D. H. Sattinger, Monotone methods in nonlinear elliptic and parabolic boundary value problems, Indiana University Mathematics Journal 21 (1971/1972), 979-1000.

[12] P. Stehlík, Periodic boundary value problems on time scales, Advances in Difference Equations 2005 (2005), no. 1, 81-92.

[13] S. G. Topal, Second-order periodic boundary value problems on time scales, Computers \& Mathematics with Applications 48 (2004), no. 3-4, 637-648.

Petr Stehlík: Department of Mathematics, University of West Bohemia, Univerzitní 22,

Plzeň 306 14, Czech Republic

E-mail address: pstehlik@kma.zcu.cz 